\title{
ORIGINAL ARTICLE Long-term effect of MRI on sacral anterior root stimulator: the Stoke Mandeville experience
}

\author{
L Lopez de Heredia, TMM Meagher, MA Jamous and RJ Hughes
}

Study design: Retrospective review study.

Objectives: To identify whether it is safe to perform a magnetic resonance imaging (MRI) exam on patients who have a sacral anterior root stimulator (SARS).

Setting: Adult patients with spinal cord injury and implanted SARS attending the National Spinal Injuries Centre who have had MRI scans.

Methods: Retrospective review between 1989 and 2010. The effect of the MRI scans on the function of the SARS (Finetech-Brindley, UK) was assessed up to 6 months following MRI at 0.2 and 1.5T.

Results: A total of 18 patients with SARS implants had MRI scans at 0.2 and $1.5 \mathrm{~T}$ of the cervical, thoracic and lumbar spine, head, shoulder and pelvis. MRI examinations were abandoned on two occasions in one patient due to radiofrequency interference. One patient's stimulator ceased to function 5 months following MRI; both of these patients showing complications had their MRI examinations at $0.2 \mathrm{~T}$. There were no adverse effects at $1.5 \mathrm{~T}$. In one patient the SARS was removed within 6 months after MRI due to an unrelated medical condition. A total of 17 patients showed no symptoms that required terminating the examination. In all the 11 patients with a complete and functioning implant before MRI at 1.5T, the SARS was functioning appropriately, and no change in bladder function was reported up to 6 months following MRI.

Conclusion: It is safe to perform an MRI scan on patients with SARS providing the examination is conducted in a $1.5 \mathrm{~T}$ system. Spinal Cord (2012) 50, 294-297; doi:10.1038/sc.2011.135; published online 22 November 2011

Keywords: MRI; SARS; implantable devices; bladder neurostimulation; spinal cord injury

\section{INTRODUCTION}

In the UK alone an estimated 1000 people suffer from spinal cord injuries (SCI) each year. Many of those patients experience bladder dysfunction, which is a major contributor to the morbidity of SCI. In the past, renal pathology has been a major cause of death in this population group. Today, mortality has declined, partly because of the considerable progress made in bladder management. One of the methods of managing voiding dysfunction as a result of SCI is through the use of the Finetech-Brindley sacral anterior root stimulator (SARS), an implantable device that effects bladder voiding by delivering low levels of electrical stimulation to intact spinal nerve roots to elicit functional contraction of the innervated detrusor and sphincter muscles. The SARS device consists of an external controller and a transmitter block and implantable parts such as electrodes, cables and an implantable receiver-stimulator. ${ }^{1-3}$

In addition to urological problems, SCI patients are prone to developing other complications including pressure sores, ${ }^{4}$ heterotopic ossification $^{5}$ and neurological ascending deterioration including subacute progressive ascending myelopathy, ${ }^{6}$ for which they require complex diagnostic imaging and in particular magnetic resonance imaging (MRI). MRI uses powerful magnetic fields and radiofrequency pulsed waves to generate images. Currently, MRI scanning of patients with implantable devices including SARS is contraindicated in many radiology departments, although the evidence in the published literature is scant and inconclusive to support such restrictions.
There is concern that induction of current in the SARS implant during MRI examination may lead to surrounding tissue damage due to the generation of excessive heat, and displacement of the nerve stimulator from the overlying tissue leading to painful stimulation. MRI examination may also lead to an alteration in the programmed parameters, intrinsic implant failure and damage to its associated components.

Despite concerns, several hundred patients with different implantable devices have safely undergone a range of MRI exams for diagnostic purposes. ${ }^{7-10}$ Advancement in the design of implantable devices and MRI systems have contributed to a reduction in complication rates as well as increased satisfaction rates in patients with SARS implants.

Because of the current restrictive policy there is a lack of direct clinical evidence regarding the effect of MRI on bladder neurostimulators, particularly on SARS devices. In a recent study to examine implanted sacral nerve stimulators, Elkelini and Hassouna ${ }^{10}$ assessedsix patients with bladder neurostimulator who underwent a total of eight MRI examinations at $1.5 \mathrm{~T}$ of the brain, cervical and thoracic spine. No patients reported any unusual symptoms during the examination and imaging was not affected by the procedure. Additionally, no malfunctions or changes in voiding parameters were found.

The aim of this study is to determine the long-term effect of diagnostic MRI on SCI patients with implanted SARS. We describe 
our experience from the past 20 years using 0.2 and $1.5 \mathrm{~T}$ MRI scanners and we address the safety of performing MRI in this population group.

\section{MATERIALS AND METHODS}

A total of 18 patients with spinal cord lesions with an implanted FinetechBrindley sacral nerve stimulator (SARS) were subjected to 44 MRI scans. The mean number of MRI was 2.5 scans per patient, operating at static field strengths of 0.2 and $1.5 \mathrm{~T}$, between 1989 and 2010. In all, 23 MRI examinations were performed at 0.2 and 21 at $1.5 \mathrm{~T}$. The majority of the MRI examinations were performed on the cervical and thoracic spine (70\%) but also on the brain, knee, pelvis, shoulder and lumbar spine. The mean period between implantation of SARS devices and patients' first MRI examination was 8.9 years.

All patients had clinical indications for MRI examination requested by the spinal consultants for diagnostic purposes. The risks of MRI examination were discussed with the patients and informed consent obtained. Patients were retrospectively reviewed during and after MRI examination. Clinical notes were examined for any reported adverse events during MRI exams. Additionally, the function of the implantable devices, changes in the stimulation parameters and voiding variations were also reviewed for a minimum period of 6 months following the MRI procedure.

\section{RESULTS}

In all, 18 patients were reviewed, 12 were male and 6 female. The average age at the first MRI was 46 years (range 24-69 years). The average time post injury at first MRI was 14 years (range 2-29 years) and the average time between injury and SARS implantation was 7 years (range 1-18 years). Of the 18 patients, 9 had a single procedure following SARS implantation. The other 9 patients underwent on average 4 scans with a combined total of 35 MRI examinations. Out of the 35 MRI procedures, 6 were conducted in 3 patients that had an incomplete SARS device with residual parts left in situ. These 3 patients were previously scanned successfully without any reported incidents $2-$ 5 years before the SARS stopped being used. Reasons for discontinuation of the device were due to causes unrelated to MRI scanning, involving surgery and removal of the receiver block or cables. One of the patients stopped using the SARS within 6 months after MRI examination as a result of an ilial conduit surgery due to transitional cell carcinoma of the bladder. A year later, the same patient underwent an MRI of the pelvis with the non-working SARS implant (Table 1). No abnormal symptoms were reported during or after imaging.

Two MRI examinations conducted on a same patient at $0.2 \mathrm{~T}$ in different years had to be stopped due to interference with the SARS device giving rise to toe movements. A subsequent MRI at $1.5 \mathrm{~T}$ was successfully performed without any complications during imaging and without later changes in bladder function (Table 1).

One patient reported complications with the SARS' hardware 5 months following MRI at $0.2 \mathrm{~T}$. During the review of the device it was noted with the help of X-ray that the receiver block was folded. One of the connectors was lying over instead of beside the receiver. The non-working SARS was adjusted after a week. The same patient had a previous MRI performed without incidents and four additional MRI examinations at $0.2 \mathrm{~T}$ without any adverse effects after resolution of the SARS' technical issues (Table 1).

In all, 13 patients had an MRI at $1.5 \mathrm{~T}$ but in 2 of these cases the SARS implant was incomplete without a receiver block. None of the 11 patients with a functional and complete SARS implant having 18 MRI examinations operating at $1.5 \mathrm{~T}$ from 2000 to 2010 reported any symptoms or discomfort that required stopping the undergoing scan. None of these patients reported any adverse effects, changes in the programmed parameters in any SARS device tested or changes in bladder function up to a minimum of 6 months following MRI investigation.

\section{DISCUSSION}

MRI has become an essential diagnostic imaging test for patients with SCI due to its high spatial and contrast resolution. Hundreds of SCI patients with bladder dysfunction have implantable SARS devices to manage this complication. This patient population typically requires MRI examinations for various clinical indications.

Despite the guidance of expert medical physicists ${ }^{11,12}$ and the general view of MRI as a safe, non-invasive and important diagnostic tool current policy in most radiology departments stipulates that MRI is contraindicated for patients with implantable devices including bladder neurostimulators.

There are several aspects that raise concern regarding the effect of MRI on patients with implantable SARS devices. The potential risk to the effective functioning and safety of bladder neurostimulators is based on the forces from the static magnetic eld, radiofrequency field, as well as electrical magnetic fields generated by the MRI scanner. The static magnetic field, which is constantly present, even when the MRI scanner is not imaging, can attract ferromagnetic components such as iron, cobalt and nickel from implantable devices.

This attraction force may cause displacement of the implantable device inside the body by twisting it into alignment with the magnetic field. ${ }^{13}$

Previous studies have shown that for magnetic fields up to $1.5 \mathrm{~T}$, the magnetic force and torque on implantable devices will be less than the force and torque due to gravity of the earth $\left(9.81 \mathrm{~N} \mathrm{~kg}^{-1}\right) .{ }^{14-16}$ Presently, no complaints of pain or a sensation of pulling during an MRI procedure conducted on implantable devices like pacemakers or SARS have been reported. ${ }^{10,17,18}$

The static magnetic field may affect the receiver block but not the extension cable or the electrode contacts of the SARS, which consist of platinum and iridium. Previous research demonstrated that electrode contacts made of iridium and platinum are not ferromagnetic and are thus safe for MRI examination. ${ }^{19}$

In this study none of the patients reported any pain or torque effect and only one patient had complications with the hardware following an MRI at $0.2 \mathrm{~T}$. No change in bladder function was documented immediately after the MRI procedure and the SARS device ceased functioning 5 months following imaging. It was reported that one of the connectors was lying over the internal receiver: normally the connector should be lying next to the receiver. The SARS device was subsequently repaired and the patient underwent successfully four additional MRI scans at $0.2 \mathrm{~T}$ within 3 years. Therefore it is unlikely that the malfunction detected initially in the bladder neurostimulator could have been a detrimental effect caused by the MRI procedure.

Previously it has been mentioned that no significant interaction between the magnetic field and the implantable device occurs when the device does not enter the magnet bore, decreasing the safety concern. ${ }^{15}$ In this study no adverse events were documented after MRI of three patients having incomplete SARS devices with residual cables and electrodes left in situ. Furthermore, one of these patients underwent an MRI examination of the pelvis, which was conducted above the remaining components.

The radiofrequency field that is used for the acquisition of MR images may lead to the induction of current in the extension cables and electrodes of implantable devices. There is a risk that some of this energy may pass through the electrode contact points into surrounding tissue. Because of the limited conductivity of the tissue, the energy can be converted into heat at the tip of the electrodes. The MRI specific absorption rate calculated in Watts $\mathrm{kg}^{-1}$, measures the amount of radiofrequency energy absorbed by the body and it is used to predict temperature changes in implantable devices. For MRI scans it 
Table 1 MRI characteristics and effect on the survival of function of SARS implants

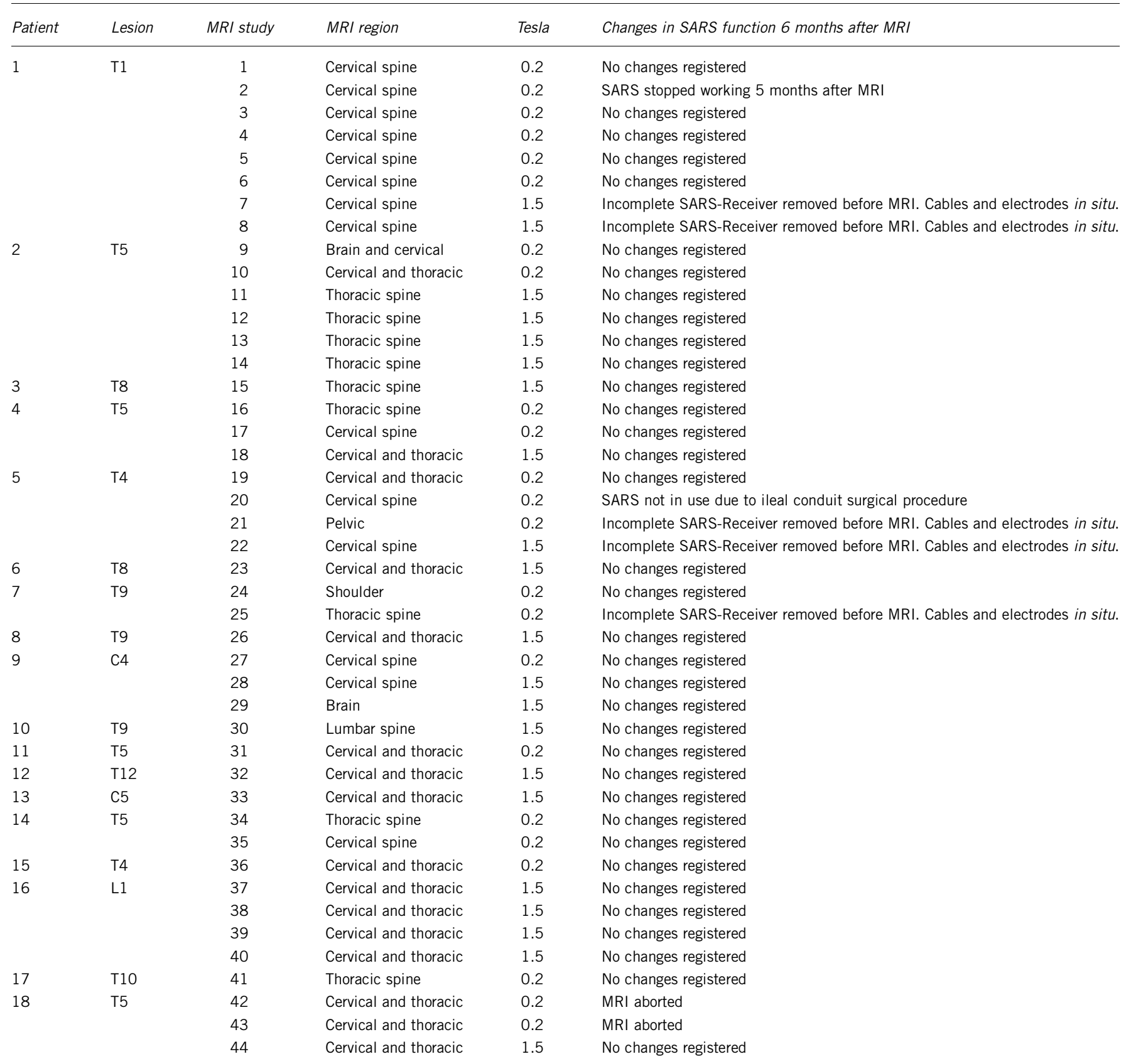

Abbreviations: MRI, magnetic resonance imaging; SARS, sacral anterior root stimulator.

is recommended by the manufacturer that the specific absorption rate should not exceed 1.1 Watts $\mathrm{kg}^{-1}$ for a scan of $30 \mathrm{~min}$.

Beside the additional factors can influence the amount of heating generated during MR imaging including the location and condition of the cables and the neurostimulator. The manufacturer advises that the function of each electrode should be tested before MRI scanning. Imaging a patient with a broken implanted lead may result in excessive heating around the break in the lead.

In this study, no complications involving heating were reported. Conversely, 2 MRI examinations conducted at $0.2 \mathrm{~T}$ were stopped due to radiofrequency interference. According to the manufacturer this is not surprising because the radio frequency of a $0.2 \mathrm{~T}$ scanner is
8.4 MHz, close to the frequency to which the Finetech-Brindley receivers are most sensitive.

The same patient was subsequently MRI scanned successfully in a $1.5 \mathrm{~T}$ scanner with no complications.

There was no radiofrequency interference or adverse effects reported in any MRI conducted in a $1.5 \mathrm{~T}$ scanner. These results agreed with a previous study where no effects were observed in patients with SARS after MRI examination at $1.5 \mathrm{~T}^{10}$

The main limitation of this study resides in its retrospective design. Limitations inherent to retrospective reviews include a dependency on previously recorded data that maybe limited. For instance, because all the MRI examinations were performed due to a clinical need, several 
technical aspects were not recorded. Additionally, due to the current contraindicative policies for MRI in patients with implantable devices, our analysis was conducted on a limited number of patients.

\section{CONCLUSION}

On the basis of over 20 years of experience at the National Spinal Injuries Centre, we conclude that MRI examination conducted at $1.5 \mathrm{~T}$ is safe to perform in patients with implantable Finetech-Brindley SARS devices when it is indispensable for diagnosis and for planning treatment. However, without further studies proving otherwise a contra-indication for MRIs in patients with any active medical implant remains the presence of a failed device or broken lead wires.

\section{DATA ARCHIVING}

There were no data to deposit.

\section{CONFLICT OF INTEREST}

The authors declare no conflict of interest.

1 Brindley GS. History of the sacral anterior root stimulator, 1969-1982. Neurourol Urodyn 1993; 12: 481-483.

2 Brindley GS. The sacral anterior root stimulator as a means of managing the bladder in patients with spinal cord lesions. Baillieres Clin Neurol 1995; 4: 1-13.

3 Brindley GS. The first 500 patients with sacral anterior root stimulator implants: general description. Paraplegia 1994; 32: 795-805.

4 Ruan CM, Escobedo E, Harrison S, Goldstein B. Magnetic resonance imaging of nonhealing pressure ulcers and myocutaneous flaps. Arch Phys Med Rehabil 1998; 79: $1080-1088$

5 Wick L, Berger M, Knecht H, Glucker T, Ledermann HP. Magnetic resonance signal alterations in the acute onset of heterotopic ossification in patients with spinal cord injury. Eur Radiol 2005; 15: 1867-1875.
6 Planner AC, Pretorius PM, Graham A, Meagher TM. Subacute progressive ascending myelopathy following spinal cord injury: MRI appearances and clinical presentation. Spinal Cord 2008; 46: 140-144.

7 Loewy J, Loewy A, Kendall EJ. Reconsideration of pacemakers and MR imaging. Radiographics 2004; 24: 1257-1267.

8 Gimbel JR, Johnson D, Levine PA, Wilkoff BL. Safe performance of magnetic resonance imaging on five patients with permanent cardiac pacemakers. Pacing Clin Electrophysiol 1996; 19: 913-919.

9 Martin ET, Coman JA, Shellock FG, Pulling CC, Fair R, Jenkins K. Magnetic resonance imaging and cardiac pacemaker safety at 1.5-Tesla. J Am Coll Cardiol 2004; 43: 1315-1324.

10 Elkelini MS, Hassouna MM. Safety of MRI at 1.5Tesla in patients with implanted sacral nerve neurostimulator. Eur Urol 2006; 50: 311-316.

11 Shellock FG. Vocare bladder system, implantable functional neuromuscular stimulation. In: Reference Manual for Magnetic Resonance Safety, Implants, and Devices: 2007 EDITION, 3rd edn. Biomedical Research Publishing Company: Los Angeles, CA, 2007, pp 311-315.

12 Fine Tech Medical Ltd,. MRI Guidance Instruction for FineTech-Brindley Bladder Stimulator. In: Document Reference: TF1-BS-402, 2008 (http://www. finetech-medical. co.uk/LinkClick.aspx?fileticket=s\%2bs1cG57Z4k\%3d\&tabid=95\&language=en-GB).

$13 \mathrm{Ng} \mathrm{K-H}$, Faust O, Acharya UR. Health effects and safety of magnetic resonance imaging. J Med Syst 2010 (doi: 10.1007/s10916-010-9460-9).

14 Baker KB, Nyenhuis JA, Hrdlicka G, Rezai AR, Tkach JA, Shellock FG. Neurostimulation systems: assessment of magnetic field interactions associated with 1.5- and 3Tesla MR systems. J Magn Reson Imaging 2005; 21: 72-77.

15 Luechinger R, Duru F, Scheidegger MB, Boesiger P, Candinas R. Force and torque effects of a 1.5-Tesla MRI scanner on cardiac pacemakers and ICDs. Pacing Clin Electrophysiol 2001; 24: 199-205.

16 Roguin A, Zviman MM, Meininger GR, Rodrigues ER, Dickfeld TM, Bluemke DA et al. Modern pacemaker and implantable cardioverter/defibrillator systems can be magnetic resonance imaging safe: in vitro and in vivo assessment of safety and function at $1.5 \mathrm{~T}$. Circulation 2004; 110: 475-482.

17 Sommer T, Vahlhaus C, Lauck G, von SA, Reinke M, Hofer U et al. MR imaging and cardiac pacemakers: in-vitro evaluation and in-vivo studies in 51 patients at $0.5 \mathrm{~T}$. Radiology 2000; 215: 869-879.

18 Duru F, Luechinger R, Candinas R. MR imaging in patients with cardiac pacemakers. Radiology 2001; 219: 856-858.

19 Tronnier VM, Staubert A, Hahnel S, Sarem-Aslani A. Magnetic resonance imaging with implanted neurostimulators: an in vitro and in vivo study. Neurosurgery 1999; 44: $118-125$. 